\title{
Introduction to the special issue: Overview of OLYMPUS Optimization Benchmark Challenge
}

\author{
R. M. Fonseca ${ }^{1}$ - E. Della Rossa ${ }^{2}$ - A. A. Emerick ${ }^{3}$ R. G. Hanea ${ }^{4}$ - J. D. Jansen ${ }^{5}$ \\ Received: 9 September 2020 / Accepted: 9 September 2020 / Published online: 13 November 2020 \\ (C) The Author(s) 2020
}

Keywords Field development optimization · Uncertainty $\cdot$ Drainage strategy optimization $\cdot$ Benchmark challenge $\cdot$ OLYMPUS · Robust decision support

Numerical optimization has been shown to be a potentially valuable technology to provide decision support in various stages of the life cycle of hydrocarbon fields. Over the past decade, many studies have appeared on numerical optimization of well controls such as rates, pressures, and ICV settings. These studies have investigated the optimization algorithms, handling of uncertainty, constraints and multiple objective functions, and more recently also various measures of risk. In recent years, the application of optimization methods has been extended more and more frequently to the field development stage during which decisions are made that have a significant impact on the performance of the field. The types of

\section{J. D. Jansen}

j.d.jansen@tudelft.nl; https://www.tudelft.nl/jd jansen

R. M. Fonseca

Rahul.Fonseca@tno.nl

E. Della Rossa

ernesto.dellarossa@eni.com

A. A. Emerick

aemerick@gmail.com

R. G. Hanea

rhane@equinor.com

1 TNO Expertise Group for Petroleum Geosciences, NL-2595, DA The Hague, The Netherlands

2 Eni, Via Emilia 1, 20097, San Donato Milanese, Italy

3 Petrobras Research and Development Center, Av. Horácio de Macedo 950, Cidade Universitária, Rio de Janeiro, RJ 21941-915, Brazil

4 Research \& Technology Center Rio, Equinor, Rua do Russel 804, Glória, Rio de Janeiro, RJ 22.210-010, Brazil

5 Faculty of Civil Engineering and Geosciences, Delft University of Technology (TU Delft), 2600, GA Delft, The Netherlands controls that have been considered have been extended to also include various field development parameters such as well positions and trajectories and, more recently, drilling schedule. Some of these controls have even been considered jointly. While for typical well controls, a number of approaches have emerged as more promising than others, for field development-type problems, no clear consensus has emerged yet. Additionally, very rarely do the various available studies use the same set of models or objective function definitions. This makes consensus generation difficult as the results from the various studies are case-dependent. One mechanism to achieve consensus on best practices and applicability of algorithms is through benchmark comparison studies. This is because the set of models, problem definition, and objective function calculation is standardized making the comparison of the results a fair process.

The potential of numerical optimization was demonstrated in a 2008 benchmark comparison study, SPE Applied Technology Workshop on Closed Loop Reservoir Management (CLRM) held in Brugge, which included the optimization of well controls [1]. CLRM is the combination of well control and/or field development optimization with computer-assisted history matching to keep the optimization models "evergreen" using the most recent production data. All benchmark participants were asked to perform both a history match and an optimization step, thus completing the full loop once. One drawback of the closed-loop setting was that different participants generated different history matched models which were subsequently used for optimization exercises, using different approaches. As a result, the relative contributions from history matching and optimization steps were somewhat difficult to disentangle. Additionally, it turned out to be difficult to draw firm conclusions on the relative performance of different algorithms used in the individual steps 
(i.e., history matching or optimization) since only the data mismatch and the final outcome were evaluated and compared. Furthermore, the 2008 closed-loop Brugge benchmark study assumed a fixed field development with a given set of wells and completions. The development of the field in terms of number, type, and placement of wells was not part of the optimization while this may be expected to have a major impact on the value that can be realized. A more detailed review of the Brugge benchmark study was provided by Peters et al. [2], who also discuss some other relatively minor issues.

The first step of any reservoir management workflow would ideally be an optimization of the field development plan. There is therefore clear value in comparison benchmarks for history matching and/or optimization separately. Many insights were gained by the reservoir optimization community as a result of the Brugge benchmark exercise. New research-focus areas were pursued, and many new algorithms have been developed for a variety of problems since 2008. Thus, a comparison of different approaches would be very useful to assess the applicability of methods for field development optimization under geological uncertainty. This motivated the launch of a benchmarking exercise for field development optimization under uncertainty. Note that history matching does not form part of the current benchmark which is only addressing well control and field development optimization given an ensemble of reservoir models.

In the remainder of this editorial, we delve into the scope of this benchmark exercise and provide a description of the geological characteristics of the model. The definition of the objective function calculation and all associated operational constraints are also provided. Finally, we end with the problems defined to be tackled using numerical optimization techniques and a brief review of the different methods and control parameterizations used in the context of this benchmark challenge and the special issue.

\section{Benchmark scope and criteria}

In the development of a benchmark study, it is essential to clearly define the criteria to be followed in order to set up a benchmark exercise. In this benchmark study, we utilize the following criteria, as suggested by Peters et al. [2], to develop an ensemble of reservoir models suitable for field development optimization:

1. Sufficiently challenging (i.e., no trivial optimal solution) to distinguish between approaches and methods and identify strengths and weaknesses

2. Realism of property distributions, uncertainty, constraints, and number/types of decision variables

3. Relatively short simulation time to allow evaluation of techniques in a manageable time frame
4. Clearly defined outcome measures such that results can be compared.

The benchmark study is aimed at field development (FD) optimization under uncertainty. Questions that we aim to address in this study are:

1. What added value can be expected if optimization methods would be applied to make field development decisions?

2. What are good workflows to arrive at optimal development plans?

3. Which controls should be considered to construct an optimal development plan?

4. Which methods are best suited for field development optimization?

5. Should well placement and control be considered jointly?

These questions can be answered by formulating and addressing a number of well-defined challenges that are detailed further below. The scope as defined above introduces the need to address several technical issues that have not been part of previous benchmark studies:

- Some controls may naturally appear as integer or binary variables. For example, well positions and drilling ordering are often thought to require treatment as integer controls.

- The number of wells may not be constant throughout an optimization process.

- Incorporating drilling costs into the objective function, and considering the well drilling order, will lead to nonsmoothness of the objective function.

- Evaluating different well trajectories requires frequent recomputation of well-reservoir connectivity.

- Joint well placement and control optimization is a mixedcontrol problem that is expected to be challenging for some workflows and algorithms.

- The FD problem will involve time-dependent nonlinear input and/or output constraints that could be handled by the simulator or, more formally, by an optimizer.

The criteria and scope listed above are about the problem definition and the associated challenges with field development optimization. Another aspect integral to the challenge is to define geological characteristics to be incorporated within the ensemble of models to be used for the optimization.

\section{Geological model characteristics and uncertainty}

The following geological features were identified as sources of complexity to be included in a reservoir model: 
- Vertical barrier - one completely sealing vertical barrier to flow poses the question of how to best develop two separate reservoirs with the same set of wells.

- Faulting - makes regular well patterns suboptimal, i.e., placement of individual wells needs to be optimized.

- Fault throws - should be such that the upper reservoir zone is juxtaposed against the lower reservoir zone at places.

- Fluvial channels - at least in one of the reservoir zones. They introduce the challenge of high-connectivity and fast water breakthrough (undesired), as well as the possibility to tap into multiple good sand with a single extended reach well.

- $K_{v} / K_{h}$ ratio - a very low vertical permeability $K_{v}$ (relative to the horizontal permeability $K_{h}$ ) makes vertical wells the only reasonable option. The $K_{v} / K_{h}$ ratio should therefore not be too low.

- Inclined layering - tends to suppress the recovery, especially in combination with a low $K_{v} / K_{h}$, as parts of the field will remain unswept.

\section{OLYMPUS benchmark model: geological description}

A synthetic reservoir model, OLYMPUS, inspired and loosely based on Brent-type oil fields in the North Sea, was developed for the purpose of the proposed benchmark study for field development optimization. The field is $9 \mathrm{~km}$ by $3 \mathrm{~km}$ and is bounded on one side by a fault with a large throw. In addition to the boundary fault, six smaller, internal faults are present in the reservoir. The reservoir is $50 \mathrm{~m}$ thick and consists of two zones that are separated by an impermeable shale layer. The upper reservoir zone contains fluvial channel sands embedded in floodplain shales. The lower reservoir zone consists of alternating layers of coarse, medium, and fine sands that are inclined with respect to the general structural dip of the field, so-called clinoforms.

The model consists of grid cells of approximately $50 \mathrm{~m} \times$ $50 \mathrm{~m} \times 3 \mathrm{~m}$ each. The model has 341,728 grid cells of which 192,750 are active. Four different facies types have been modeled in the different layers. An overview of the different facies types with their properties is provided in Table 1.

Petrophysical properties such as porosity, permeability, and net-to-gross (NTG) were generated using standard geostatistical techniques for the different facies types. No porosity-permeability relationship has been used, based on the assumption that insufficient data are available at the early stage of field development. The permeability values in the $X$ and $Y$ directions are identical. The permeability in the $Z$ direction is $10 \%$ of the permeability in the $X$ direction. From the available exploration well logs, the depth of the oil-water contact (OWC) was determined to be at $2092 \mathrm{~m}$, with an in situ hydrostatic pressure of 205 bar. The initial water saturation distribution is modeled using capillary pressure curves. Different capillary pressure curves were assigned to the different facies types. Thus, changes in the facies model will lead to different initial water saturation distributions. The dead oilbased fluid properties used in the ensemble of models are given in Table 2. Note that oil viscosity is modeled as a function of pressure and hence, a range is provided in Table 2. Similarly, the oil formation volume factor is also provided as a function of pressure.

\subsection{Model realizations}

An ensemble of 50 realizations was generated wherein the facies are regenerated by altering the random seed. The grid geometry, fault geometries, and oil-water contact are considered to be known for this case and are therefore the same in all realizations. Thus, the uncertain properties are:

1. Facies

2. Porosity

3. Permeability

4. Net-to-gross ratio

5. Initial water saturation

6. Transmissibility across the faults

Upscaled permeability fields for four different realizations for layer 3 are illustrated in Fig. 1. The orientation and number of channels vary in the top reservoir section while in the bottom reservoir section, the clinoformal stratigraphic sequence is varied as illustrated in Fig. 2.
Table 1 Facies types and property distribution ranges for the OLYMPUS reservoir models

\begin{tabular}{lllll}
\hline Facies type & Zones present & Net-to-gross & Porosity range & Permeability range $(\mathrm{mD})$ \\
\hline Channel sand & Upper & $0.8-1$ & $0.2-0.35$ & $400-1000$ \\
Shale & Upper \& Barrier & 0 & 0.03 & 1 \\
Coarse sand & Lower & $0.7-0.9$ & $0.2-0.3$ & $150-400$ \\
Medium sand & Lower & $0.75-0.95$ & $0.1-0.2$ & $75-150$ \\
Fine sand & Lower & $0.9-1$ & $0.05-0.1$ & $10-50$ \\
\hline
\end{tabular}


Table 2 Fluid properties of the OLYMPUS reservoir models

\begin{tabular}{llll}
\hline Symbol & Variable & Value & Unit \\
\hline$C_{\text {rock }}$ & Rock compressibility & $1.42 \times 10^{-5}$ & $1 / \mathrm{bar}$ \\
$c_{\mathrm{w}}$ & Water compressibility & $3.97 \times 10^{-5}$ & $1 / \mathrm{bar}$ \\
$\mu_{\mathrm{o}}$ & Oil dynamic viscosity & $2.8-3.5$ & $\mathrm{cP}$ \\
$\mu_{\mathrm{w}}$ & Water dynamic viscosity & 0.398 & $\mathrm{cP}$ \\
$\rho_{\mathrm{o}}$ & Oil density & 850 & $\mathrm{~kg} / \mathrm{m}^{3}$ \\
$\rho_{\omega}$ & Water density & 1020 & $\mathrm{~kg} / \mathrm{m}^{3}$ \\
$d_{\text {well }}$ & Well-bore diameter & 0.19 & $\mathrm{~m}$ \\
$T$ & Simulation time & 7200 & $\mathrm{~d}$ \\
\hline
\end{tabular}

The ensemble of models was generated using the following procedure. A high-fidelity base case model of approximately 5 million grid cells was generated as a first step. Five wells were drilled into this base case model and synthetic logs were generated for each of these wells. These logs were then used to constrain the generation of the ensemble of 50 high-fidelity models to capture uncertainty. Each of these high-fidelity models was upscaled for the purpose of flow simulations using the flow-based upscaling method. The location of the oil/water contact is kept constant in all the model realizations. The ensemble of models that has been provided consists of an upscaled set of models.

\section{Drainage strategy and production volumes}

Figure 3 illustrates the impact of uncertainty in the 50 model realizations represented in terms of cumulative oil and water production as well as cumulative water injection and field water cut, for a reference operating strategy. The reference strategy consists of 11 producers and 7 injectors which are operated on a pressure constraint. The placement of the wells in this reference strategy was a result of a manual trial and error exercise based on engineering judgment for a chosen realization. Thus, the well placement strategy is probably not optimal over all the realizations. The results were obtained by running a commercial fully implicit black oil simulator [3], for each realization. As can be observed in Fig. 3, the uncertainty can be visually classified as relatively large, which can be interpreted as representative of a greenfield development scenario. Table 3 provides the minimum, maximum, and average value for the different properties plotted which can be a way to substantiate the degree of uncertainty.

This illustrates that the realizations show very different responses especially in terms of cumulative water injected and cumulative oil produced. The range in the model responses also suggests that there exists significant scope to optimize a field development plan.

\section{Optimization problem descriptions}

Three tasks/problems were defined as part of this benchmark exercise which are explained below. An optimized strategy is often compared with a reactive control strategy i.e. a shut-in of the well when its production becomes uneconomical. The economic water cut based on the prices provided in Table 4 has been estimated to be $88 \%$.

\subsection{Objective function}

The performance of the field development plan is measured by expected net present value (NPV) as evaluated over the set of 50 model realizations (i.e., mean NPV). The cost and revenue contributions for the NPV calculation are listed in Table 4.

The following formula should be used to compute the NPV for a single realization in USD (\$):

$$
N P V=\sum_{i=1}^{N_{t}} \frac{R\left(t_{i}\right)}{(1+d)^{t_{i} / \tau}},
$$

Fig. 1 The permeability in layer 3 from four different model realizations showing different realizations of fluvial channels in a shale background
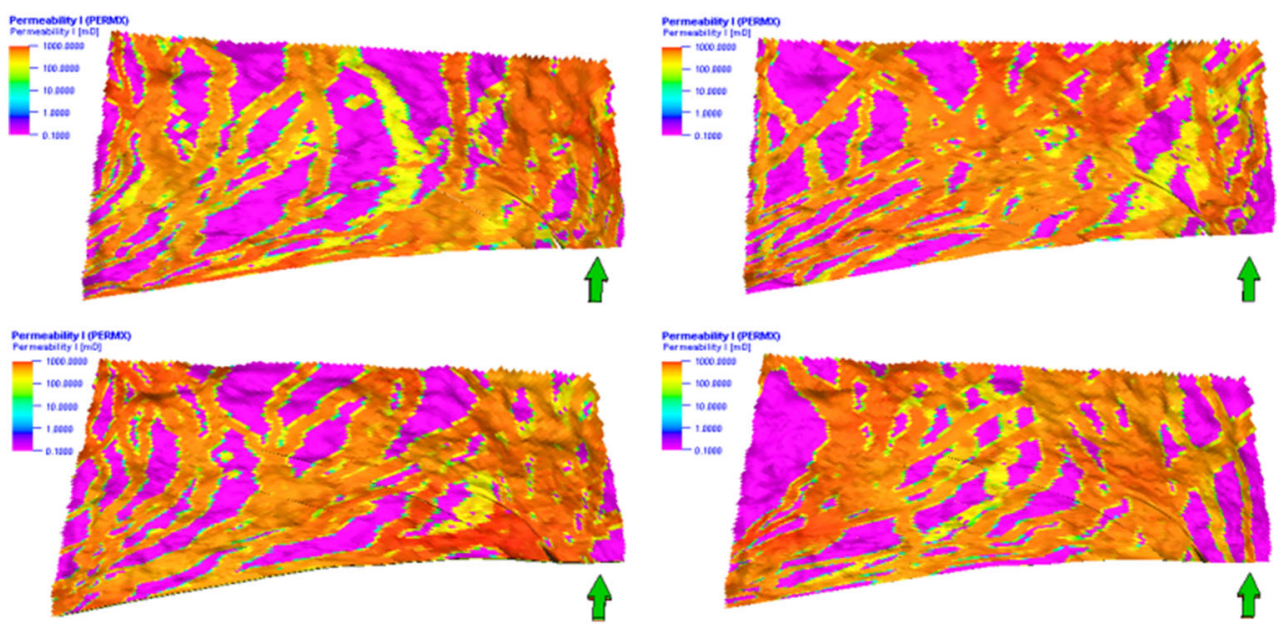
Fig. 2 The permeability in layer 13 from four different model realizations showing different realizations of the clinoformal stratigraphic sequences

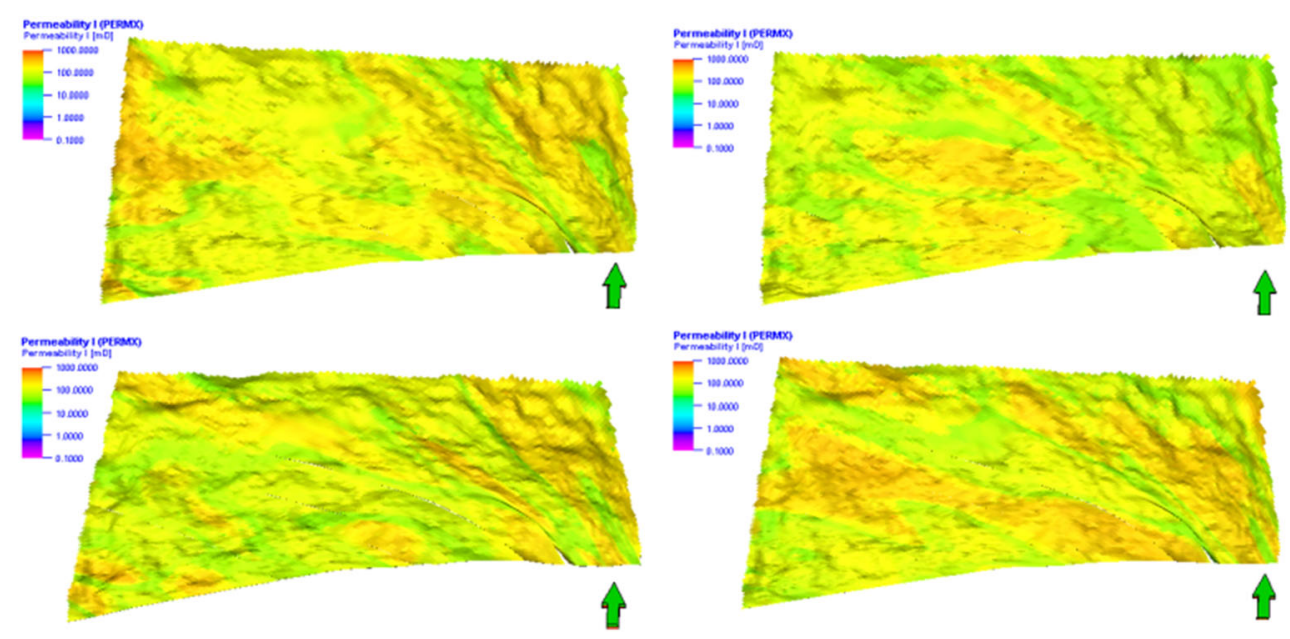

where index $i$ refers to the time interval with length $\Delta t_{i}=t_{i}-t_{i}$ ${ }_{-1}$ and starting at $t_{i-1}$ and ending at time $t_{i}$, all in days; $N_{t}$ is the total number of time intervals over the life cycle period; $d$ is the discount factor (expressed as a fraction); $\tau$ is the time interval for discounting (365 days); and $R\left(t_{i}\right)$ is the sum of all expenses and incomes incurred during the time interval $\Delta t_{i}$. The time intervals are fixed to calendar months to ensure consistency in the NPV calculation for all participants. All cash flows and discounting are assumed to take place on time $t_{i}$. Well drilling and completion costs associated with finished drilling and completion of a well in the time interval $\Delta t_{i}$ are also assumed to be incurred at the time $t_{i}$. The platform investment cost must be introduced in the time interval in which drilling of its first well starts (which is the very first month for the first platform). The cost term $R\left(t_{i}\right)$ in $\$$ is defined as

$$
\begin{aligned}
R\left(t_{i}\right)= & Q_{o p}\left(t_{i}\right) \cdot r_{o p}-Q_{w p}\left(t_{i}\right) \cdot r_{w p}-Q_{w i}\left(t_{i}\right) \\
& \cdot r_{w i}-P\left(t_{i}\right)-D\left(t_{i}\right),
\end{aligned}
$$

where $Q_{o p}\left(t_{i}\right), Q_{w p}\left(t_{i}\right)$, and $Q_{w i}\left(t_{i}\right)$ are the total oil production, water production, and water injection volumes over the time interval $\Delta t_{i}$, respectively. For example: $Q_{o p}\left(t_{i}\right)=F O P T\left(t_{i}\right)-$ $F O P T\left(t_{i-1}\right)$. Furthermore, $r_{o p}, r_{w p}$, and $r_{w i}$ are the corresponding oil revenue (price) and water production and injection costs in $\$$ per unit volume, whereas $P\left(t_{i}\right)$ is the platform-investment costs, $D\left(t_{i}\right)$ is the total well drilling, and completion costs incurred during the time interval $\Delta t_{i}$ specified in Table 4 . The operational criteria for the wells and information about the other necessary constraints for the wells are provided in Table 5 .

\subsection{Task 1: well control optimization}

The aim of this exercise is to perform well control optimization. A development plan with 18 wells is provided. The location and trajectory of the wells are fixed/given for this exercise. The well targets/controls can be adjusted every 3 calendar months (i.e., at the first day of every third month) Participants are free to choose control time intervals as any multiple of a 3-month period. For the 18 wells in the deck and a life cycle period of 20 years, this would result, in the case of 3-month control intervals, in a total of 1440 controls as all the wells are assumed to be drilled and completed at the starting time. Flexibility in the choice of the total number of controls which is a function of the number of control time intervals is provided. Furthermore, the choice of the well controls to be optimized, e.g., rates, pressures, and single well PI multipliers, is left to the discretion of a user. The optimal strategies must adhere to the bounds on well flow rates and bottom-hole pressures specified in Table 5 as well as the maximum liquid production rate specified.

\subsection{Task 2: field development optimization}

The aim of this task is to optimize a field development plan. The number, type, order, and trajectories of the wells as well as the platform location to be used are the focus of the optimization. The well placement strategy provided for the well control exercise does not need to be used.
Table 3 Volumetric and simulation results for a given development strategy for OLYMPUS reservoir models

\begin{tabular}{llll}
\hline Property & Maximum value & Minimum value & Average value \\
\hline Cumulative oil produced & $14.50{\text { million } \mathrm{m}^{3}}^{3}$ & 7.42 million $\mathrm{m}^{3}$ & 11.12 million $^{3}$ \\
Cumulative water injected & 64.78 million $\mathrm{m}^{3}$ & 19.17 million $\mathrm{m}^{3}$ & 42.22 million $^{3}$ \\
Field water cut & $94 \%$ & $83 \%$ & $90 \%$ \\
\hline
\end{tabular}


Fig. 3 The cumulative oil produced (top left), water produced (top right), water injected (bottom left), and field water cut (bottom right) for all the 50 model realizations
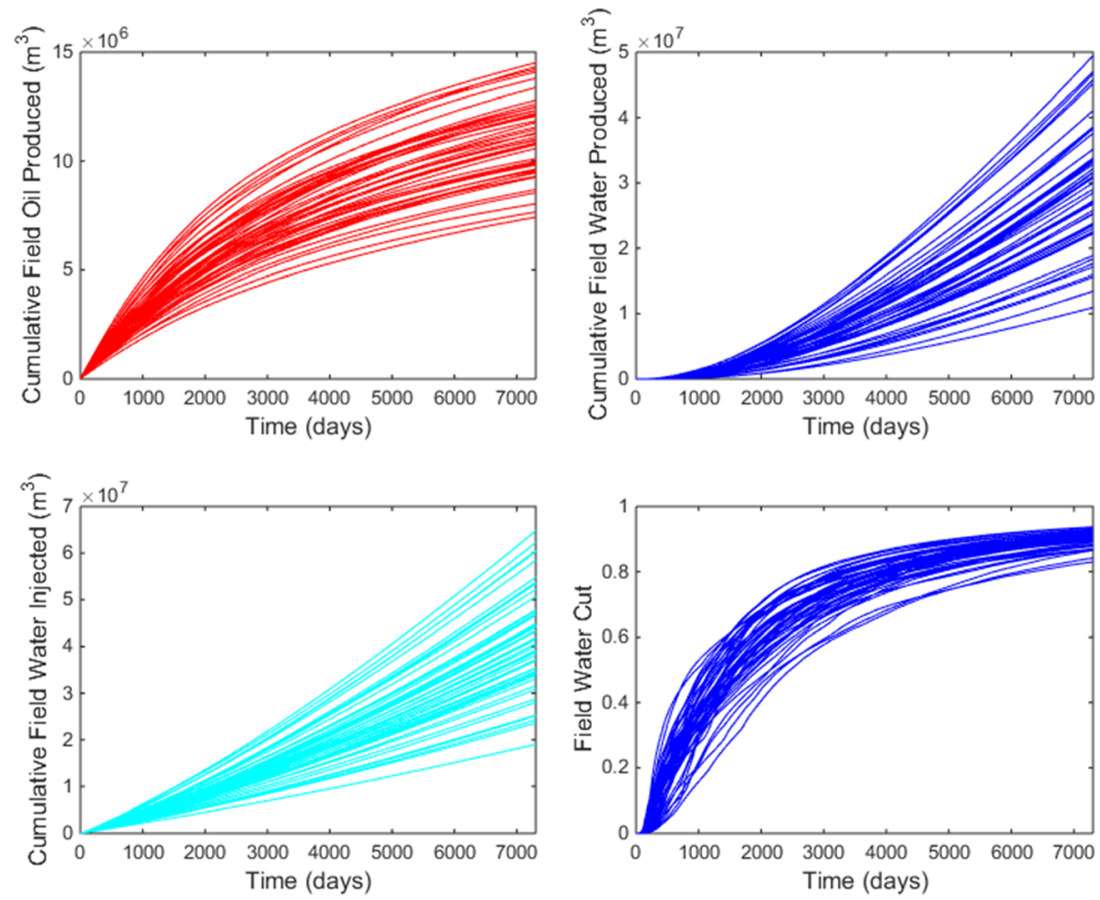

For this task, participants are expected to deliver a development plan that consists of:

- The coordinates $\left(X_{p}, Y_{p}, 0\right)$ of one or more platforms,

- A well drilling sequence (which also determines the number of wells $N_{w}$ to be drilled),

- The full trajectories (as survey files) of all drilled wells starting from a kick-off point with coordinates $\left(X_{p}, Y_{p}\right.$, $\left.Z_{k, i}\right)$ where $i=1, \ldots, N_{w}$ up to the end point of each well with coordinates $\left(X_{p}+\Delta X, Y_{p}+\Delta Y, Z_{e, i}\right)$,

- Assignment of the type of each well (producer or injector).

The field development options are constrained by a number of factors:

- The wells must adhere to a constraint on dogleg severity as applied to a smooth well-path.

Table 4 Cost and revenue inputs for calculation of the objective function

\begin{tabular}{lll}
\hline Contribution & Value & Units \\
\hline Platform investment & 500 & Million USD \\
Drilling and completion & $5000 \cdot \Delta Z+10000 \cdot|\Delta X Y|$ & $\mathrm{USD}, \mathrm{m}$ \\
Oil price & 45 & $\mathrm{USD} / \mathrm{bbl}$ \\
Cost produced water & 6 & $\mathrm{USD} / \mathrm{bbl}$ \\
Cost injected water & 2 & $\mathrm{USD} / \mathrm{bbl}$ \\
Annual discount factor & 0.08 & \\
End of life cycle period & 20 & years \\
\hline
\end{tabular}

- Each well $i$, with $i=1, \ldots, N_{w}$, can have a different kickoff depth $Z_{k, i}$.

- Only single-bore wells are allowed (i.e., no sidetracks).

- Wells cannot be converted (e.g., from producer to injector or vice versa) at later time.

- A platform has space for 20 well slots only.

- The liquid processing capacity of the platform facilities limits the field production rate.

- Drilling of each next well is started immediately after finishing the previous one, i.e., without idle time between completing one well and starting the drilling of the next well.

- There are operational well rate capacity and pressure limits as specified in Table 5

- The recovery strategy is water flooding so only water can be injected.

- The diameter for all wells is assumed to be $0.1905 \mathrm{~m}$

- Each well is assumed to come on stream immediately after drilling and completion of the well is finished.

\subsection{Drilling time calculation}

The following formula should be used for the time (in days) to drill and complete a well:

$\Delta t_{D}=0.015 \cdot \Delta Z+0.02 \cdot|\Delta X Y|$,

where $\Delta Z=Z_{k, i}$ and $|\Delta X Y|=\sqrt{\Delta X^{2}+\Delta Y^{2}}$ is the horizontal offset (step-out) of the well end point from the kick-off point. Note that this assumes that the well end point is both laterally 
Table 5 Operational and drilling constraints for the wells in the OLYMPUS reservoir models

\begin{tabular}{lll}
\hline Property & Maximum value & Units (field or SI) \\
\hline Maximum number of wells on a platform & 20 & - \\
Maximum platform liquid production rate & 88,000 or 14,000 & $\mathrm{bbl} / \mathrm{day} \mathrm{or} \mathrm{m}^{3} / \mathrm{day}$ \\
Maximum well oil production rate & 5700 or 900 & $\mathrm{bbl} / \mathrm{day} \mathrm{or}^{3} /$ day \\
Maximum well water production rate & 10,000 or 1600 & $\mathrm{bbl} / \mathrm{day} \mathrm{or}^{3} / \mathrm{day}$ \\
Maximum allowable injector BHP & 235 & $\mathrm{bar}$ \\
Minimum allowable producer BHP & 150 & $\mathrm{bar}$ \\
Maximum dogleg severity & $10 / 30.48$ & $\mathrm{o} / \mathrm{m}$ \\
\hline
\end{tabular}

and in depth the furthest point from the kick-off point. With the above formula and using the values in Table 4, we obtain a drilling and completion time of 30 days for a vertical well to $2000 \mathrm{~m}$ depth and a cost of 10 million $\$$. For a well with end point at $2000 \mathrm{~m}$ depth and $2000 \mathrm{~m}$ offset from the platform, the drilling cost and time work out to 30 million $\$$ and 70 days respectively. These and other constraints are listed and quantified in Table 5.

Production platform investment costs are assumed to be related to the installed capacity and are therefore not included in the cost per drilled well. Note that since there is no time period between drilling of two wells, the drill rig is never idle, and the rig rate is assumed to be incorporated into the costs per well. Moreover, royalties and social and corporate taxes are not considered explicitly. The simulation start time corresponds to the beginning of the drilling of the first well. It is assumed that all produced associated gas is consumed or exported. We do not include a price of gas in the economic model and assume that all oil and gas processing and exporting costs are incorporated into the oil price listed in Table 4.

\subsection{Task 3: joint field development and well control optimization}

In this task, participants are encouraged to come up with optimal field development strategies as well as well control (operational strategies). All the inputs needed for this exercise are the same as the inputs used for the field development optimization task.

\section{Results overview}

In September 2018, a 1-day workshop was organized in Barcelona for the presentation of the results from the participants of the OLYMPUS challenge. This workshop was attended by over 60 people from across the globe. The program consisted of 9 oral presentations and 17 poster presentations by participants from academia, industry, and research institutes. This Special Issue is a culmination of the peer- review process for participants who submitted their contributions. This special issue, which includes 10 peer-reviewed publications, consists of contributions from authors who participated in the workshop as well as from authors who did not. A performance comparison among the proposed optimization techniques based only on the objective function value achieved would be natural. However, we do not provide quantitative comparisons and we suggest the readership to consider, in general, the proposed algorithmic innovations.

In such benchmark challenges, there are many factors which can causes differences in results for e.g. the use of different simulators, problem assumptions, and use of ensembles. Prior to the workshop, participants were requested to submit the optimal solutions obtained for the different challenges. This was for the purpose of benchmarking the results as participants to enable a structured comparison of the results obtained. A (partial) benchmarking of the results presented by the various participants at the workshop was performed by TNO. After the workshop and before (during) the peerreview process for this special issue, the majority of the participants updated their optimized strategies, and therefore, benchmarking these updated optimal strategies was unfortunately no longer feasible. Therefore, we do not provide the benchmarked results; however, we summarize an overview of methods and approaches used to tackle the problems. Two main optimization challenges, well control and field development, were tackled by various participants. Here, we provide a summary of the approaches and observations from the results at the workshop and this special issue for these two optimization challenges.

\subsection{Well control optimization challenge}

\subsubsection{Optimization methods}

For this challenge, both gradient-based and gradient-free optimization methods were used. Adjoint-based gradient and the stochastic gradient-based approaches were used. For gradientfree methods $(\mu-\lambda)$ ES, partitioned PSO and GA and variants thereof were the methods of choice. 


\subsubsection{Control parameterizations}

The choice of parameterization of the well control problem in terms of rates, pressures, or any other type of variable was left to be decided by a participant. The granularity of control intervals was also chosen by a participant with a maximum of 1440 controls in total being defined. The gradient-based optimization users generally preferred to use the maximum number of control intervals to be optimized for either the well rates or well pressures. One gradient-based optimization participant parameterized their controls in terms of well-shut-in times and a single bottom-hole pressure per well over the provided life cycle.

The gradient-free optimization users generally used fewer control intervals which leads to fewer control variables. One participant, in particular instead of optimizing a well control variable, chose to optimize the economic water cut limit for each production well and a shut-in time limit for injection wells with the flexibility to open the wells in the future if they are shut-in. This formulation is particularly interesting because it combines elements of reactive control together with proactive control and although the optimizer finds a single optimal strategy, each realization will behave very differently with this formulation.

\subsubsection{Ensemble size and computational efficiency}

The two varieties of gradient-based optimization methods were implemented using the full ensemble of 50 models during the optimization process. The gradient-free optimizations were performed with a smaller number of model realizations, usually 5 models. This reduced subset of model realizations was chosen with different approaches. Despite using a reduced subset of model realizations during the optimization process, the computational efficiency of gradient-free methods was generally much inferior compared with those of the gradient-based methods.

\subsection{Field development optimization challenge}

\subsubsection{Optimization methods}

For this challenge, most participants chose to use gradient-free methods. Only two participants used gradient-based methods wherein the stochastic gradient formulation was preferred. The adjoint-based gradient was not used by anyone to tackle this optimization problem. For gradient-free methods, GA, BHPSO, experimental design + GA, PSO, and variants thereof were the methods of choice. One paper considered a geology-based heuristic approach to field development planning instead of a numerical optimization technique.

\subsubsection{Control parameterizations}

The choice of parameterization of the platform location, well trajectory, well order, number of wells, and well types differed across the participants. Some papers in this special issue and other participants at the workshop did not optimize all the variables requested in the problem description and most did not explicitly account for the dogleg severity constraints provided. Additionally, when optimizing well trajectories, it is essential to accurately calculate the well connection factors especially for deviated and horizontal wells. These aspects were explicitly dealt with by only a selected number of participants and papers in this special issue.

A majority of papers tackle the different variables in this challenge with a pre-processing step considering expert knowledge either in the form of saturation maps to find an informed initial guess for the optimization or to select certain decision options in a staged approach to field development studies. Additionally, since the well trajectory problem can be very different from the well order problem and the number-of-well problems, some papers consider different optimization methods for the different problems in a sequential approach. One paper special issue considered most of the variables simultaneously.

The optimized number of wells and well types (producer/ injector) was similar for most of the participants. While the platform locations were similar, the well trajectories obtained were fairly different with one paper only considering vertical wells while other papers targeting different well types for the different reservoir sections. The initial guess showed some sensitivity to the optimal results for this optimization challenge.

\subsubsection{Ensemble size and computational efficiency}

Three papers used the full 50-member ensemble during the optimization process for this challenge. Some participants used a single model realization for the optimization process while other participants used either a fixed subset of model realizations or schemes which progressively change/increase the number of realizations used in the optimization process. Once again, the computational efficiency of the gradient-free methods is inferior to the gradient-based methods. Comparing the papers which have used the full 50-member ensemble for optimization, the gradient-based methods are shown to be an order of magnitude more efficient in terms of the total number of simulations performed. Naturally, there will be a tradeoff between the computational efficiency and objective function value achieved and is therefore left to the judgment of a reader to draw their own conclusions. 


\section{Post-workshop}

The OLYMPUS dataset has been downloaded by more than $100+$ organizations covering academia, research institutes, and industry. The OLYMPUS model has been used in many publications not only for field development optimization workflows but also for benchmarking simulator and solver performance, applications for machine learning-based workflows and to test cloud-based reservoir simulation solutions.

\section{Conclusions}

This editorial provides an overview of the OLYMPUS field development optimization benchmark challenge. The scope, criteria, model, and problem description are provided as well as an overview of the methods used by the participants at the workshop as well as the papers published in this special issue. We hope that this exercise will spur new ideas and, in the future, new research developments can be benchmarked to the provided data set. The data set and simulation input files for this benchmark case can be obtained via the following webpage https://www.isapp2.com/optimization-challenge/ optimization-challenge-download-files.html.

Acknowledgments The OLYMPUS benchmark study is an initiative of the partners of the Integrated Systems Approach to Petroleum Production (ISAPP) research consortium consisting of TNO, Delft University of Technology, Equinor, ENI, and Petrobras. We would like to acknowledge the contribution of Dr. Kees Geel for help with building the OLYMPUS geological models. We would like to acknowledge the contribution of Dr. Olwijn Leeuwenburgh and Dries Hegen in defining the scope and organizing the OLYMPUS benchmark challenge, respectively. We would like to thank all the reviewers who made this special issue possible and the authors who submitted their manuscripts for publication.

Open Access This article is licensed under a Creative Commons Attribution 4.0 International License, which permits use, sharing, adaptation, distribution and reproduction in any medium or format, as long as you give appropriate credit to the original author(s) and the source, provide a link to the Creative Commons licence, and indicate if changes were made. The images or other third party material in this article are included in the article's Creative Commons licence, unless indicated otherwise in a credit line to the material. If material is not included in the article's Creative Commons licence and your intended use is not permitted by statutory regulation or exceeds the permitted use, you will need to obtain permission directly from the copyright holder. To view a copy of this licence, visit http://creativecommons.org/licenses/by/4.0/.

\section{References}

1. Peters, L., Arts, R.J., Brouwer, G.K., Geel, C.R., Cullick, S., Lorentzen, R.J., Chen, Y., Dunlop, K.N.B., Vossepoel, F.C., Xu, R., Sarma, P., Alhuthali, A.H., Reynolds, A.C.: Results of the Brugge benchmark study for flooding optimization and history matching. SPEREE. 13(3), 391-405 (2010). https://doi.org/10. 2118/119094-PA

2. Peters, E., Chen, Y., Leeuwenburgh, O., Oliver, D.S.: Extended Brugge benchmark case for history matching and water flooding optimization. Comput. Geosci. 50, 16-24 (2013). https://doi.org/ 10.1016/j.cageo.2012.07.018

3. Eclipse. 2017. https://www.software.slb.com/products/eclipse

Publisher's note Springer Nature remains neutral with regard to jurisdictional claims in published maps and institutional affiliations. 